\section{Cover illustration: Not just another pretty face}

The Union Jack butterfly (Delias myisis) is found along the tropical northeast coast of Queensland, Australia, including the Cape York Peninsula, and has the typical languid and random flight of most butterflies. But the behaviour of these solitary creatures belies their dynamic physiology, surprising learning abilities, and truly sensational visual mechanisms.

Investigators seeking further understanding of the visual mechanisms found members of a closely related species to be quick studiers when learning the whereabouts of food. Moreover, through behavioural techniques, these same investigators proved that butterflies definitely have colour vision, and even have colour constancy. This ability allows the neurological system of butterflies to draw conclusions about colour (and food sources) when the same wavelength may be not presented. For example, a flower that appears red in broad daylight may appear to be a dark maroon on an overcast day and/or when blooming in the shade of a tree. Any animal that relies on colour perception for food must have some degree of colour constancy or would not be able to recognise food sources in differing light. As with many other insects, butterflies also see into the ultraviolet, and can even detect polarised light. Many butterflies have inborn preferences for certain colours. Although not evaluated in many butterfly species, some species are known to have preferences for yellow and/or blue.

Usually when you see a butterfly, though, you don't think of colour constancy. We are usually captivated by the gentle beauty of the creature, but did you ever consider how that colourful pattern appears? These spectacular colours are constructed from a mosaic of individual scales. Each scale cell will reliably produce a specific pigment to create the appearance of a smooth coloured surface, and this pattern is reliably placed in the pupal stage although the pigments are synthesised and deposited later, when the scales have formed. These colours are not structural, but are true pigments. The colours are important for species recognition, for warning to predators, and sometimes for camouflage.

But these beautiful wings are used for more than flight or camouflage. Taxonomically, we rely on morphological characteristics for classification, but recent evidence suggests there may be other methods. The valvae and wings of adult males of various Delias species have been documented to produce volatile compounds which are species specific and are probably important in mate attraction for these solitary insects. In fact, these compounds are so specific that chemotaxonomic classification is possible, suggesting another mechanism at tracing evolution, or even classification.

As with most insects, butterflies have compound eyes. These optical sampling devices are quite different from any of the vertebrate ocular systems, and usually fit the animal's ecological needs, as suggested in a previous cover (BFO, March 2001).

This species, as do other butterflies, has an appositional compound eye, but with an unusual twist. (By official classification there are at least eight types of eyes: camera, concave mirror, pinhole, appositional, neural superpositional, refracting superpositional, reflecting superpositional, and parabolic superpositional, although more types are possible depending on classification system, and each type has its own visual mechanisms.) An appositional eye does not resolve the image beyond what each facet perceives directly. The array of ommatidia in an appositional compound eye produces good sensitivity to movement, but acuity can be limited by diffraction and limited capture of photons; hence, this eye performs best in a daylight environment - or perhaps only in a daylight environment. In this species, the cornea (this term refers to the first surface that light encounters as it enters the ommatidium) acts as a lens that helps to focus light on the rhabdom and, in essence, each ommatidium corresponds to a single rod or cone in the vertebrate retina. Rhabdoms are invertebrate photoreceptors which consist of individual retinular cells or rhabdomeres, either fused or separate, which combine to create a cylindrical rod-like structure that fills the distal half or two thirds of the ommatidium.

Ommatidia have an impressive array of modifications to satisfy the ecological needs of each species. For example, the direction of the face of each ommatidium can be determined and compared to adjacent faces or other ommatidia to see the extent of the visual field. Maps of the direction of faces of ommatidia have not been completed for many species, but those that have been mapped prove interesting. In some species, there is a central region that has the optical axes tilted more or less towards one another which are essentially parallel or at least having a reduced angle from the neighbouring ommatidium creating a portion of the visual field that has greater sampling. This, then, is a foveal equivalent in an invertebrate compound eye.

Other ommatidial adaptations have been found that assist in light reflection or concentration. For example, some diurnal butterflies have a thick pebbling across the surface of the cornea. These nipple-like projections have the dimensions of $1 / 2 \lambda$ in the centre of the visible spectrum and create an aspheric surface, thus giving external constructive interference and avoiding reflection and creating greater absorption of light. This adaptation is especially useful for nocturnal lepidoptera to maximise light collection, but is present in diurnal butterflies and other insects for camouflage. Interestingly enough, this adaptation betokened a similar use for this technology in antireflective coating of certain commercial optics

Butterflies do something even more exotic with their visual system and this is the special twist. They have a structure in the proximal tip of each ommatidial cone as a second element that acts to create an extraordinary lens system. The crystalline structure of this second element (following the "cornea" as mentioned above) has a focal power of $200000 \mathrm{D}$ ! This marvellous adaptation within the ommatidial cone, when combined with the corneal lens, creates a minute Keplerian telescope with magnification of approximately 6-7 times. As a result, this eye is described as an afocal appositional compound eye. It is afocal because the image is focused in front of the second element, and the second element recollimates the light causing it to stimulate the rhabdom as parallel rays. (Nilsson D-E, et al, Nature 1984; 312:561-3.)

Wait, there's more! Most butterflies have interesting tapeta, the reflecting material distal to the photoreceptors that will reflect all light or specific wavelengths of one colour, and represents an example of a biological interference filter. This structure is composed of modified tracheoles, and creates the colour by constructive and destructive interference by using layers of chitin and air with a specific distance between each layer that corresponds to a fraction of the wavelength of the specific colour of light reflected. This manner of tapetal reflection represents convergent evolution as vertebrate tapetal mechanisms are quite different. Butterflies also have been found to possess coloured pigment in close apposition to the rhabdom which probably alter the spectral sensitivity of the rhabdom.

And you thought that a butterfly was just another pretty face. -Ivan R Schrwab, MD, UC Davis Department of Ophthalmology, 4860 Y Street, Ste 2400, Sacramento, CA 95817, USA, (irschwab@ucdavis.edu). 